\title{
Earth-Moon-Sun System: Eclipses Models
}

\author{
Rosa M. Ros \\ Department of Applied Mathematics 4, Technical University of Catalonia, \\ Jordi Girona 1-3, 08034 Barcelona, Spain \\ email: ros@mat.upc.edu
}

\begin{abstract}
Everybody understands what is meant by an eclipse: a body that covers another one. However, the general audience and the students in particular do not know exactly "What the relative positions of the Earth, the Sun and the Moon have to be in order for an eclipse to take place?"

You can read a newspaper or watch TV and observe that the information about an eclipse is not correct. In my country, it was stated, in a very important newspaper, that "the solar eclipse will take place tomorrow because there will be a Full Moon".

Teaching this topic in schools or in educational sessions in observatories, it is a good idea to introduce a simple model that maintains the proportion between the diameters and distances of these three bodies. This contribution will introduce this model, showing their applications in the playgrounds of the school, for sunny days or in the classroom for cloudy days.
\end{abstract}

Keywords. eclipses, miscellaneus, history of astronomy

\section{Introduction}

The term "eclipses" is given to very different phenomena. It is not easy for a general audience and students to understand very well what this concept means. It is not easy for everybody to distinguish between a solar or lunar eclipse if we take into account the relative positions of Earth, Moon and Sun. Solar eclipses happen when the Moon is in a new phase and the Moon eclipses take place when the Moon is full.

When the teacher/speaker manages to explain this ideas and everybody seems to understand that, a new question appears: Why are there not eclipses at each new Moon or in full Moon phases. It is necessary to mention that the Moon orbit is inclined by 5 degrees to the ecliptic plane and the eclipses take place when the Moon is near the nodes line, but it looks as though this idea is very difficult to introduce to non-initiated people.

In any case it is clear that it is necessary to find a new way to explain all these concepts. A new simple way closer to ideas commonly used by the speaker. We will introduce the following simple model to do this.

\section{The Earth-Moon model}

It is possible to build a simple model to show the Moon phases and the differences between the lunar and solar eclipses Broman, Estalella \& Ros (1997). In order to make this model, it is enough to nail 2 nails (about 3 or $4 \mathrm{~cm}$ long) onto a $125 \mathrm{~cm}$-long strip of wood. The distance between both nails must be $120 \mathrm{~cm}$. On each nail we will fix a "polystyrene"sphere of 4 and $1 \mathrm{~cm}$ of diameter in order to simulate the Earth and the Moon (figure 1).

It is very important to use the size mentioned because in this case we have a scale model of the EarthMoon system according the proportions of diameters and distances (1:320000 scale). 
Table 1. Illustrates the relationships between the diameters and distances in the Earth-Moon model

\begin{tabular}{lcc}
\hline Earth diameter & $12800 \mathrm{~km}$ & $4 \mathrm{~cm}$ \\
Moon diameter & $3500 \mathrm{~km}$ & $1 \mathrm{~cm}$ \\
Earth-Moon distance & $384000 \mathrm{~km}$ & $120 \mathrm{~cm}$ \\
Sun diameter & $1400000 \mathrm{~km}$ & $440 \mathrm{~cm}=4.4 \mathrm{~m}$ \\
Earth-Sun distance & $150000000 \mathrm{~km}$ & $4700 \mathrm{~cm}=47 \mathrm{~m}$ \\
\hline
\end{tabular}

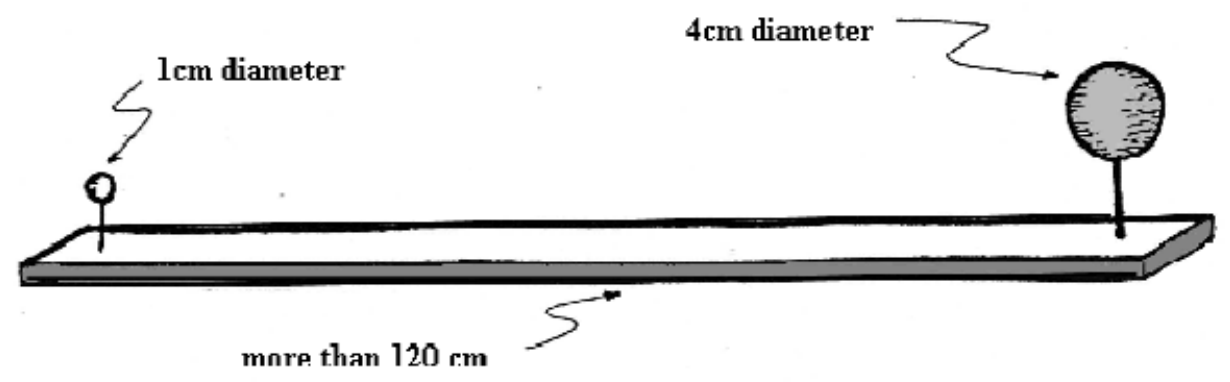

Figure 1. Model of the Earth-Moon system.

Using the model it is possible to note:

- There are only Moon eclipses when it is a Full Moon

- There are only Moon eclipses when it is a New Moon

- Solar eclipses are only visible in one small region of the Earth.

It is difficult to have the Earth and the Moon in the correct straight line position in order to produce an eclipse each time that the Moon is in its full or new phase

\subsection{Moon Phase simulation}

In a sunny place and when the Moon is visible, you have to point the small sphere (which represents the Moon in the model) in line with the real Moon in the sky, while you are holding the big sphere (the Earth) next to your face.

The observer can see the Moons (real and the sphere of the model) with the same size and the same phases. If you change the orientation of the stick you can reproduce the phases of the Moon depending on the light that it receives from the Sun. You should move the Moon from the right to the left in order to reproduce the sequence of phases.

It is better to do this activity in the playground of the school, but if it is cloudy, you can reproduce the activity inside the classroom using the overhead projector.

\subsection{Moon eclipses simulation}

In order to simulate Moon eclipses you can use the overhead projector (as the Sun) and the model (Earth-Moon system) inside the classroom. It is better to use the overhead projector to avoid looking directly into the Sun. Take the model, pointing the Earth towards the Sun, move the Moon into the Earths shadow (figure 2). The cone of shadow is bigger than the Moon and you can easily see a Moon eclipse.

\subsection{Solar eclipses simulation}

Using the overhead projector once more you can simulate a solar eclipse putting the model with the Moon in front of the overhead projector (figure 2). You can move the model in order to get the Moons shadow to appear on the Earths surface. It is not easy to achieve this. For this reason there are less solar eclipses than Moon eclipses. 

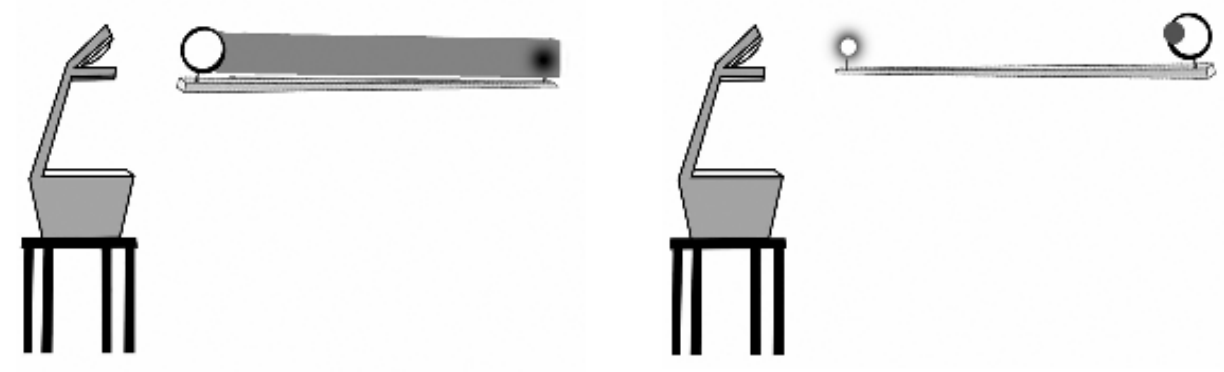

Figure 2. Lunar eclipses simulation on the left and solar eclipses simulation on the right.

If the two bodies are correctly placed in a straight line, you can see a small shadow on the white sphere of the Earth (figure 2). That means that the solar eclipses are a local phenomena only visible in one region of the Earth.

This model is very useful in order to explain the geometrical position of three bodies: Sun, Earth and Moon. After using the model for a few minutes people reach some conclusions. Everybody understands that it is easier to produce lunar eclipses than solar eclipses. They also understand that the Moon eclipses are visible for many more people that the solar ones that are only visible locally. Then everybody understands that it is normal that we can observe every year a Moon eclipse, but we have to wait a lot of years to have the opportunity to observe a solar eclipse in our country.

Moreover in the previous model the idea of how a small Moon can cover the Sun 400 times bigger than our satellite is missing. In order to explain that it is good to introduce the following model.

\section{The Sun-Moon model}

In order to visualize the Sun-Earth-Moon system with special emphasize on distances, we will consider a new model taking into account the terrestrial point of view of the Sun and the Moon. In this case we will invite the students to draw and paint a big Sun of $280 \mathrm{~cm}$ diameter(of almost 3 meters diameter) on a sheet and we will show them that they can cover this with a small Moon of $0.7 \mathrm{~cm}$ diameter (less than $1 \mathrm{~cm}$ diameter). It is a good idea to substitute the Moon ball for a hole in a wooden table in order to be sure about the position of the Moon and the observer.

It is very important to use the size mentioned because we are using 1:500000 scale for the SunMoon model in order to maintain the proportions of diameters and distances (table 2).

In this model, the Sun will be fixed 300 meters away from the Moon and the observer will be at $77 \mathrm{~cm}$ from the Moon. The students feel very surprised that they can cover the big Sun with this small Moon. Really this relationship of 400 times is not easy to imagine. It is good to show them with an example in order to understand the scale of distances and the real sizes in the universe. All these exercises and activities help them (and may be us) to understand which are the spatial relationships between celestial bodies during a solar eclipse. This method is much better than reading a series of numbers in a book.

Perhaps, it is a good idea to complement this model including Mercury and Venus between the Earth and Sun. But take into account that if you also want to place Mars, Jupiter and Saturn, you should put them at $450 \mathrm{~m}$, at $1.5 \mathrm{~km}$ and, almost, $3 \mathrm{~km}$ far from the Sun Fucili, Garcia, Casali(1999).

\section{Activities using eclipsis observations}

Eclipses were a special opportunity for the humanity in order to discover new knowledge about the Sun-Earth-Moon system. We can promote these events to students in order that they get also some information. Of course the kind of activities should be 
Table 2. Illustrates the relationships between the diameters and distances in the Sun-Moon model

\begin{tabular}{lcc}
\hline Earth diameter & $12800 \mathrm{~km}$ & $2.55 \mathrm{~cm}$ \\
Moon diameter & $3500 \mathrm{~km}$ & $0.7 \mathrm{~cm}$ \\
Earth-Moon distance & $384000 \mathrm{~km}$ & $77 \mathrm{~cm}$ \\
Sun diameter & $1400000 \mathrm{~km}$ & $280 \mathrm{~cm}$ \\
Earth-Sun distance & $150000000 \mathrm{~km}$ & $300 \mathrm{~m}$ \\
\hline
\end{tabular}

different according to the age and education of the students. In some cases there are some opportunities to carry out very sophisticated activities. We will introduce in the following section the method developed by Aristarcho in order to study the distances and diameters of Sun-Earth-Moon system. But for some students that did not use trigonometry we will present some very simple activities to get information without too much difficulty.

\subsection{Lunar eclipses}

The lunar eclipses take place when the Moon crosses the Earths shadow. And the EarthSun distance is very big, we can consider that the solar rays are practically parallel when they get to Earth, and the size of our planet and its shadow are practically the same.

Using 3 or 4 photos made during a lunar eclipse in the correct position at the time of the eclipses, we can observe the shadow limit. If students draw a series of circles (with diameters in intervals of $0.5 \mathrm{~cm}$ ) on a piece of card and cut them. After several tries, they can find the "best card adapted to the shadow.

They can measure the diameter of the Moon (on the photos) and the Earth (on the card). Using the real value of the terrestrial diameter, by means of a proportion they can obtain the diameter of our satellite (figure 3).

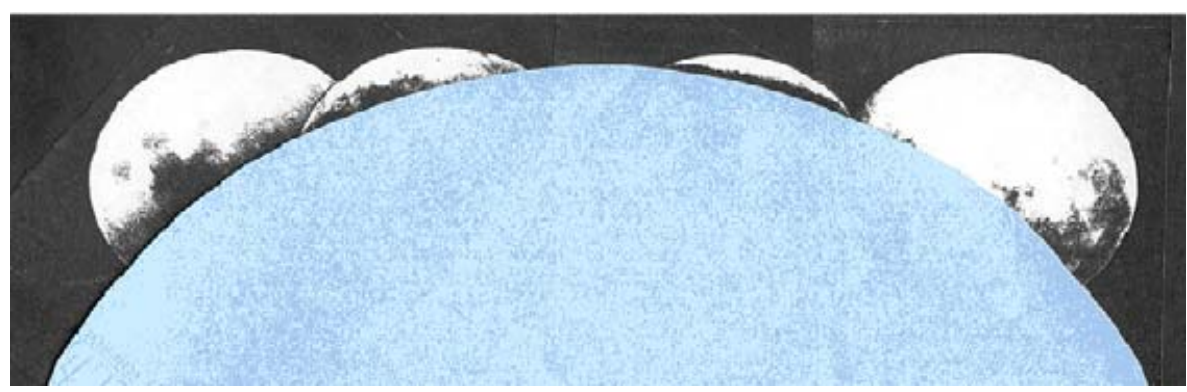

Figure 3. Lunar photos and terrestrial card.

\subsection{Solar eclipses}

The lunar eclipses take place when the Moon crosses the Earths shadow. It is not unusual to observe solar eclipses by means of a "camera obscura". We can use them in order to calculate the diameter of the Sun. In any case we can produce a "camera obscura" with a carton cylinder that students can get from central axis from a roll of aluminium paper or transparent paper in the kitchen at home or a shoes box.

We will use one of the mentioned cylinders. We will cover one of the ends with semitransparent millimetre squared paper and the other one with aluminium paper. We produce a small hole using a needle in the aluminium paper end (figure 4).

It should place the cylinder part with the hole directed towards the Sun and to observe the other part with millimetre squared paper. We can measure the diameter $d$ of the Sun on the millimetre squared paper. 


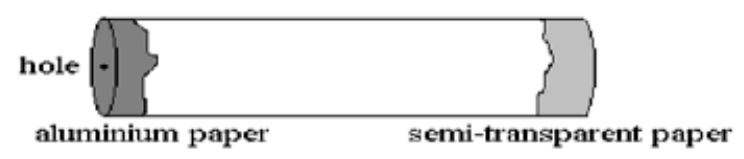

Figure 4. Preparing the camera obscura device.

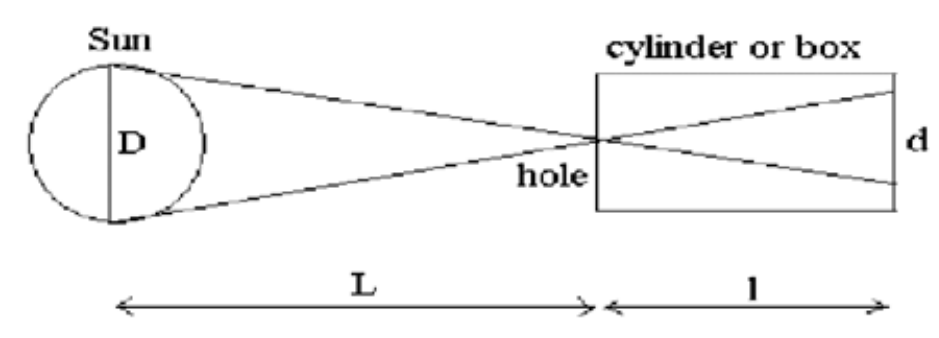

Figure 5. Geometrical proportions in order to calculate the Sun's diameter.

In order to calculate the Sun diameter we consider figure 5. We can taking into account two triangles in the previous figure, and we introduce the relationship:

$$
D=\frac{d L}{l}
$$

Of course, it is necessary to introduce the distance $L$ Earth-Sun in the formula in order to get the solar diameter. This is the reason that this method does not seem very genuine because we have to give students this distance. But if we then want them to calculate all the values for themselves, it is necessary to use more mathematical contents such as these which we introduce in the following section.

\section{Aristarcho's experiment redone}

Aristarchos (310-230 B.C.) took into account observations of the eclipses in order to deduce some proportions between the distances and the radii of the Earth-Moon-Sun system. He calculated the Sun's radius, the Moon's radius, the distance Earth-Sun and the distance Earth-Moon concerning the Earth's radius. Some years after Eratosthenes (280-192 B.C.) determined the radius of our planet and it was possible to calculate all the distances and radii of the Earth-Moon-Sun system. The proposal of this section is to repeat both experiments with students adapting the mathematical process designed by Aristarchos Ros (1996) and, if possible to repeat the observations.

\subsection{Relationship between the Earth-Moon and Earth-Sun distances}

Aristarchos determined that the angle from the Earth to the Sun and the Moon which is in the quarter phase position was $87^{\circ}$ (figure 6 ).

We know that he made a mistake, possibly because it was very difficult for him to determine the instant of the quarter phase. In fact this angle is $89^{\circ} 51$, but the process used by Aristarchos is correct. In figure 5, if we use the definition of the sinus, it can be deduced that

$$
\sin 9^{\prime}=\frac{E M}{E S}
$$

where $E S$ is the distance from the Earth to the Sun, and $E M$ is the distance from the Earth to the Moon. Then approximately,

$$
E S=400 E M
$$

(although Aristarchos deduced $E S=19 E M$ ). 


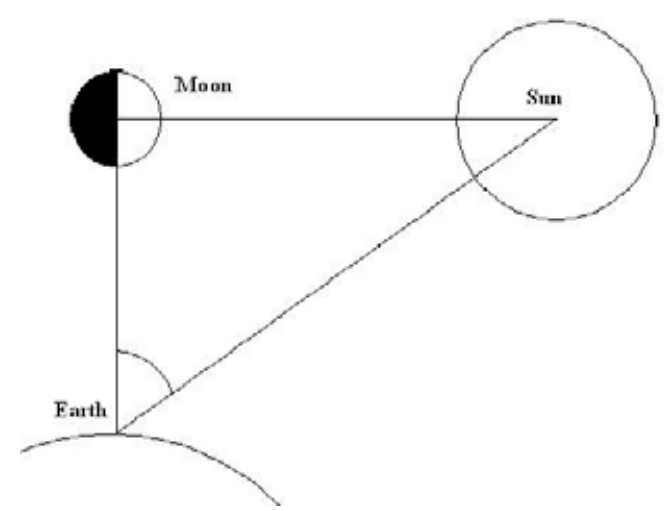

Figure 6. Moon's relative position in the quarter phase.

\subsection{Relationship between the radius of the Moon and the Sun}

The relationship between the diameter of the Moon and the Sun must be similar to the previous formula, because from the Earth we observe both diameters equal to $0.5^{\circ}$. Therefore the radius also verifies

$$
R s=400 R m
$$

5.3. Relationship between the Earth-Moon's distance and the radius of the Moon or the Earth-Suns distance and the radius of the Sun

Given that the Moon's diameter observed is $0.5^{\circ}$, with 720 times this diameter it is possible to cover the circular path of the Moon around the Earth (we assume that the Moon orbit is circular). The longitude of this path is $2 \pi$ times the distance Earth-Moon, that is to say $2 R m 720=2 \pi E M$, isolating,

$$
E M=\frac{720 R m}{\pi}
$$

and by a similar reasoning,

$$
E S=\frac{720 R s}{\pi}
$$

5.4. Relationship between the Earth's distances, the Moon's radius, the Sun's radius and the radius of the Earth

During a Moon's eclipse, Aristarchos observed that the time necessary for the Moon to cross the cone's shadow of the Earth was double the time necessary for the Moon's surface to be covered (figure 7). Therefore he deduced that the shadow of the Earth's diameter was double the Moon's diameter, that is to say, the relation of both diameters or radii was $2: 1$. Indeed we know that this value is $2.6: 1$.

Then in figure 7 we deduce the following relationship

$$
\frac{x}{2.6 R m}=\frac{x+E M}{R e}=\frac{x+E M+E S}{R s}
$$

where $x$ is an auxiliary variable. 


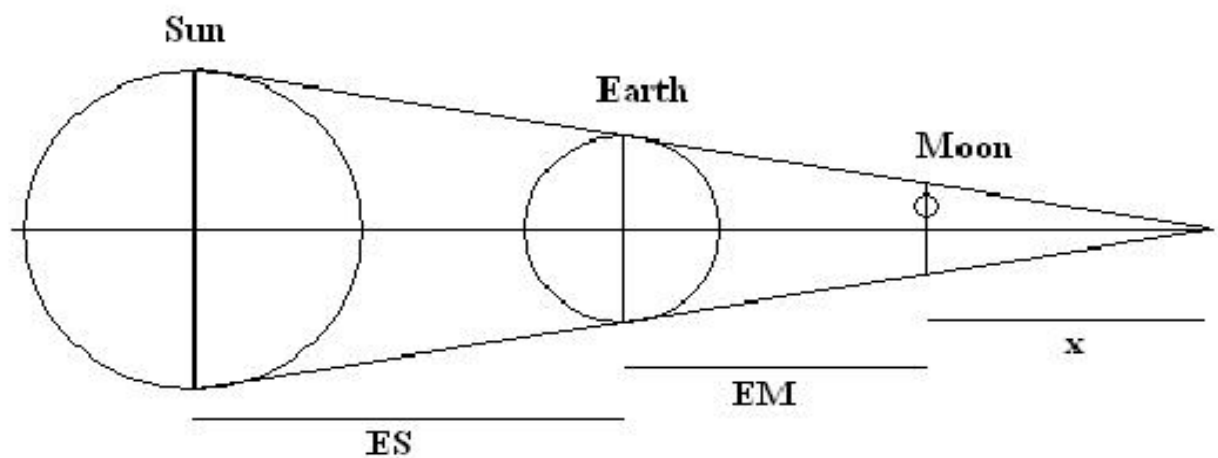

Figure 7. Relative position of Earth-Moon-Sun system in the shadow cone.

Introducing in this previous expression the relationships $E S=400 E M$ and $R S=$ $400 R M$, it is possible to eliminate $x$ and simplifying it,

$$
R m=\frac{401}{1440} R e
$$

is obtained, which offers the opportunity to express all the dimensions concerning the Earth's radius, so

$$
\begin{aligned}
& R s=\frac{2005}{18} R e \\
& E S=\frac{80200}{\pi} R e \\
& E M=\frac{401}{2 \pi} R e
\end{aligned}
$$

We only need to calculate the Earth's radius to obtain all the distances and radii of the Earth-Moon-Sun system.

\subsection{Measurements with students}

It is a good idea to repeat the measurements made by Aristarchos with students Ros (2005). In particular, the students first have to calculate the angle from the Earth, between the Moon's quarter and the Sun. For this measurement it is only necessary to use a horizontal goniometer (or theodolit or a small refractor telescope) and to find out the exact instant of the quarter phase. So we verify if this angle is $87^{\circ}$ or $89^{\circ} 51^{\prime}$ (it is not really possible for the students to achieve this precision, but the main idea is that they make the observations in order to understand the process very well).

Secondly, during a lunar eclipse, using a chronometer, it is possible to calculate the relationship of times between: "the first and the last contact of the Moon with the Earth's shadow", that is to say, measuring the diameter of the cone's shadow of the Earth (figure 8) and "the time necessary to cover the Moon surface", that is to say the Moon's diameter (figure 8). Finally it is possible to verify if the relationship between both is $2: 1$ or 2.6:1 (in this case the time observations measurements made by students are, in general, very good).

The most important objective of this activity is not the result obtained. The most important thing is to show to students that if they use their knowledge and intelligence, they can obtain interesting results with reduced facilities. In this case the ingenuity of Aristarchos was important in obtaining some concepts of the size of the Earth-Moon-Sun system.

It is a good idea to measure the Earth's radius with students according to the process used by Eratosthenes. As Eratosthenes' experiment is very well-known (see for example Ros \& Vinuales 1999), and it is not necessary to introduce it here. 


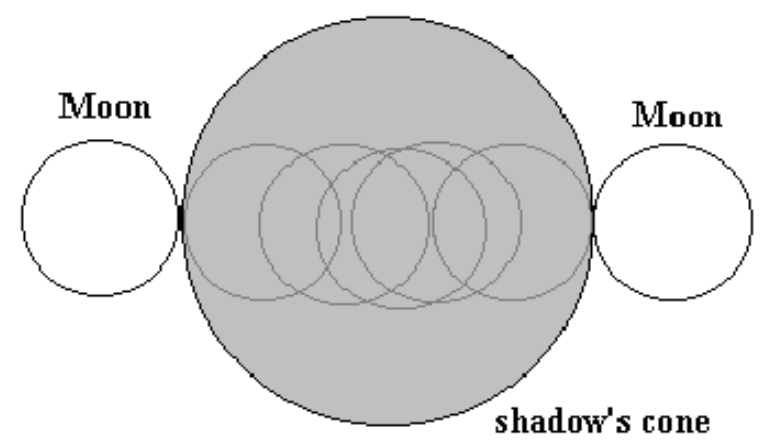

Figure 8. Measuring the shadow cone diameter.

\section{Making a "Sheetscope" to simulate the eclipses movement}

Finally we introduce a simple activity for school. If you have the collection of drawing or photos made by students, you can glue each one in a page of a spiral exercices book according to the following rules (figure 9).

(a) Cut the drawings or photos and put a number on each one (it is important not to change the order).

(b) Glue each drawing or each photo on the pages of the spiral exercices book (according the correct order).

(c) Pass the pages quickly and you can see a simulation of eclipses movement.

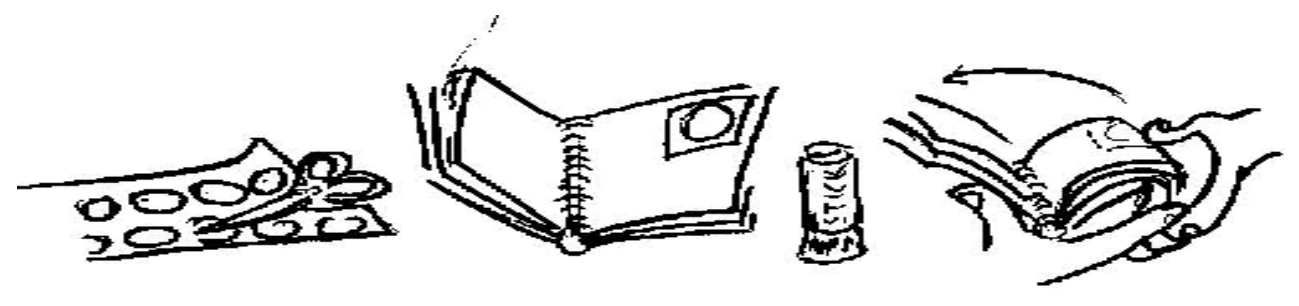

Figure 9. Using the "sheetscope" in order to simulate solar or lunar eclipses movement. Young students enjoy it a lot.

\section{Conclusions}

- Students understand eclipses: geometrical position and distances and diameters relationships

- Students found relationships within sizes of Earth-Moon-Sun system

- Students verify that observing and thinking about the dates they got, they can obtain more information about the universe

- Students learn and enjoy astronomy

\section{References}

Broman, L., Estalella, R. \& Ros, R.M. 1988, Experimentos de Astronomia Ed. Alhambra Fucili, L., Garcia, S. \& Casali, G., 1999, Proceedings 3rd EAAE Summer School, 107

Ros, R.M. 1996, J. Investigacion e Innovacion en Educacion Matematica 2, 125

Ros, R.M. 2005, Proceedings 9th EAAE Summer School, 135

Ros, R.M. \& Vinuales, E. 1999, Proceedings 3rd EAAE Summer School, 55 